\title{
On the flow topology inside droplets moving in rectangular microchannels
}

\author{
Shaohua Ma, $\ddagger^{a}$ Joseph M. Sherwood, ${ }^{b, c}$ Wilhelm T. S. Huck ${ }^{a, d}$ and Stavroula Balabani $*^{b}$ \\ Received (in $X X X, X X X)$ Xth $X X X X X X X X X 20 X X$, Accepted Xth $X X X X X X X X X 20 X X$ \\ ${ }_{5}$ DOI: 10.1039/b000000x
}

The flow topology in moving microdroplets has a significant impact on the behaviour of encapsulated objects and hence on applications of the technology. This study reports on a systematic investigation of the flow field inside droplets moving in a rectangular microchannel, by means of micro-particle image velocimetry ( $\mu$ PIV). Various water/oil (w/o) fluid mixtures were studied in order to elucidate the effects 10 of a number of parameters such as capillary number $(\mathrm{Ca})$, droplet geometry, viscosity ratio and interfacial tension. A distinct change in flow topology was observed at intermediate $C a$ ranging from $10^{-3}$ to $10^{-1}$, in surfactant-laden droplets, which was attributed primarily to the viscosity ratio of the two phases rather than the Marangoni effect expected in such systems. W/o droplet systems of lower inner-to-outer viscosity ratios tend to exhibit the well-known flow pattern characterised by a parabola-like profile in the

15 droplet bulk-volume, surrounded by two counter rotating recirculation zones on either side of the droplet axis. As the viscosity ratio between the two phases is increased, the flow pattern becomes more uniform, exhibiting low velocities in the droplet bulk-volume and higher-reversed velocities along the w/o interface. The $\mathrm{Ca}$ and droplet geometry had no effect on the observed flow topology change. The study highlights the complex, three-dimensional (3D) nature of the flow inside droplets in rectangular 20 microchannels and demonstrates the ability to control the droplet flow environment by adjusting the viscosity ratio between the two phases.

\section{Introduction}

Droplet-based microfluidics has evolved as an important platform in many aspects of scientific research, due to its ability to isolate 25 targets from their surroundings through (most commonly) w/o interfaces. Understanding of the flow pattern inside the droplets is required for successful application of the technology in, for example, chemical synthesis ${ }^{1-4}$ and single cell screening applications. ${ }^{5-7}$ A topology characterised by strong recirculation 30 zones enhances mixing, which is advantageous when studying fast reactions in droplets. On the other hand, high-throughput cell manipulation requires encapsulation at high speed, which might generate a high shear environment inside the droplets and could potentially be harmful to the viability of certain cells. ${ }^{8,9}$

35 In recent years, the dynamics of microdroplet flows have been quantified using $\mu \mathrm{PIV}$, which is an established optical method to characterise microscale flows by tracking the motion of particles seeded in the flow. ${ }^{10,11} \mathrm{~A}$ number of such studies on the droplet flow topology of liquid-liquid systems can be found in the 40 literature. ${ }^{12-17}$ For example, Oshima et al. ${ }^{13}$ applied confocal $\mu$ PIV to measure the flow field inside a moving droplet; by acquiring the flow field at various planes they were able to reconstruct the 3D flow topology using continuity. The results highlighted the role of the flow surrounding the droplet and the 45 liquid-liquid interface on the flow topology. They subsequently extended their technique to simultaneously characterise the internal and surrounding flow by using multicolour illumination. $^{18}$ Both liquid phases were also measured simultaneously by Miessner et al. ${ }^{16}$ using conventional $\mu \mathrm{PIV}$; the 50 two phases were discriminated from the captured images as only one excitation wavelength was employed and the 3D topology was also reconstructed from multiple planes.

The emphasis in the above studies was on characterising the recirculation flows and the extent of mixing, which is important 55 in applications involving reactions, synthesis or extraction for a particular fluid system. However, the flow in a droplet moving in a rectangular microchannel depends on a large number of parameters such as the $C a$, viscosity ratio between the two phases, droplet geometry and the presence or absence of a 60 surfactant. Very few studies have concentrated on the effects of such parameters on flow topology or reported on distinct flow topology changes. King et al. ${ }^{15}$ observed, through a $\mu$ PIV study, a change in the flow topology of aqueous droplets moving in Teflon tubing. By altering the length of the plugs and the bulk ${ }_{65}$ flow velocity, the strength of the internal circulation varied proportionally. Labrot et al. ${ }^{19}$ studied the effect of a wide range 
of parameters on hydrodynamic resistance and observed significant dependencies on droplet volume, viscosity and velocity at a fixed $\mathrm{Ca}$; however no attempt was made to characterise the flow field in detail. More recently, Jakiela et al. ${ }^{20}$ 5 reported a discontinuous transition in the convective velocity of droplets moving in a rectangular micron-sized channel with $\mathrm{Ca}$ for viscosity ratios less than unity. $\mu$ PIV measurements revealed that the transition was associated with a change in flow topology from one characterised by two strong recirculation regions, to one 10 exhibiting four additional counter-rotating rolls at the caps. The authors stressed the importance of characterising such changes in topology and called for the development of new theories to describe the complex flow behaviour of droplets in rectangular channels.

15 The present study probes the flow topology inside w/o microdroplets moving in a rectangular microchannel. Different droplet-carrier fluid mixtures were employed in order to elucidate the effects of viscosity ratio, droplet geometry, flow rate and surfactant (interfacial tension) on droplet flow topology.

\section{${ }_{20}$ Materials and Methods}

\section{Microfluidic device fabrication and droplet generation}

All chemicals were purchased from Sigma-Aldrich (UK), unless noted otherwise and used without further purification. Deionised water was used in all aqueous phases. Microfluidic 25 polydimethylsiloxane (PDMS) devices were fabricated by combined photo- and soft lithography. ${ }^{21}$ Detailed channel architectures were designed in AutoCAD (AutoDesk, USA) and standard photolithography techniques were used for fabrication as described by Bauer et al. ${ }^{22}$ The channel dimensions are shown in 30 Fig. 1. A channel depth of $150 \mu \mathrm{m}$ was achieved by spin-coating SU8 2025 (MicroChem, USA) at $75 \mu \mathrm{m}$ onto a 3-inch silicon wafer (Compart Technology Ltd, UK), according to the protocol supplied by MicroChem, including preheat and soft bake steps, twice. After UV light exposure in an MJB4 mask aligner (Suss 35 MicroTec, Germany), the master was hard baked for $30 \mathrm{~min}$ and developed in 1-methoxy-2-propyl acetate (Sigma-Aldrich). A commercially available PDMS kit (Sylgard 184, Dow Corning Co.) was poured on top of the master, at a mixing ratio of 10:1 w/w pre-polymer to cross-linker. After degassing and curing, the ${ }_{40}$ PDMS cast was cut, peeled off the master, punched for inlets and outlets, and bonded to a glass microscope slide. The width of the channels was $100 \mu \mathrm{m}$ upstream of the droplet formation nozzle and $200 \mu \mathrm{m}$ downstream. The channel surface hydrophobicity was modified by flushing the channel with $0.5 \%(\mathrm{v} / \mathrm{v})$ Trichloro $45(1 H, 1 H, 2 H, 2 H$-perfluorooctyl) silane in HFE7500 and incubating at $110{ }^{\circ} \mathrm{C}$ for $1 \mathrm{~h}$. The flow was delivered to the microchannel using a high-precision syringe pump (Fusion 400, Chemyx Inc., USA) via PE tubing (Becton Dickinson, USA).

Droplet illumination and image acquisition were carried out in 50 the middle region of the $200 \mu \mathrm{m}$ wide and $20 \mathrm{~mm}$ long straight channel, downstream of the droplet formation nozzle. This channel length was chosen to ensure that a steady flow was reached at the imaged region. In cell encapsulation applications channel widths and depths in the range of $50-100 \mu \mathrm{m}$ are 55 typically employed; however, larger channel dimensions enable more detailed visualization of the droplet internal flow and greater spatial resolution; hence a relatively larger geometry was employed in the present study. The volume flow rates employed in the present study varied from $10-5000 \mu \mathrm{l} / \mathrm{hr}$. In this range, the 60 Bond number, $B o=\Delta \rho g l^{2} / \gamma$, which represents the ratio of gravitational to surface tension forces, is much less than unity ( $\Delta \rho$ is the difference in fluid densities, $g$ is the acceleration due to gravity, $l$ is a characteristic length scale, and $\gamma$ is the interfacial tension between the two fluids). Thus, the flow physics were not 65 altered by using a relatively larger microchannel. The aqueous and oil phases were injected independently into the microchannels and the aqueous fluid formed droplets at the crossjunction by flow-focusing with the oil fluid. The aqueous phase was seeded with $1.1 \mu \mathrm{m}$ Nile Red fluorescent tracing particles 70 (FluoSphere F-8819, Invitrogen Inc., USA) at a concentration of $6 \times 10^{6}$ particles per $\mathrm{ml}$ before flow delivery.

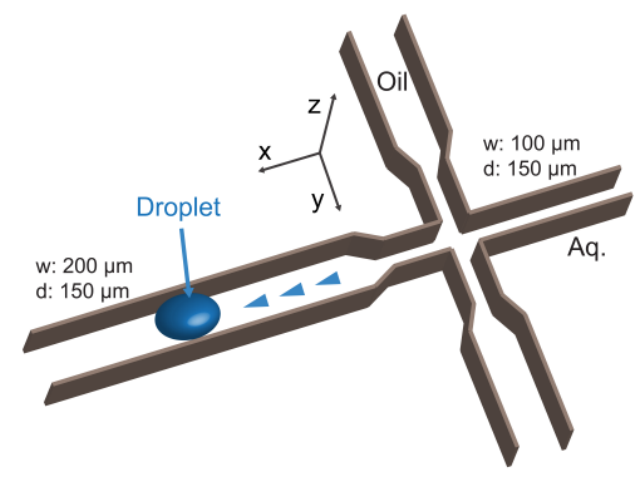

Fig. 13D drawing of the microfluidic channel architecture. The channel had a fixed depth of $150 \mu \mathrm{m}$; the channel width at the 75 imaging region, i.e. downstream of the droplet generation nozzle, was $200 \mu \mathrm{m}$. The straight channel from the nozzle to the outlet was $20 \mathrm{~mm}$ long.

Initially, a liquid-liquid system involving the use of 45/55 v/v water/glycerol (G5516, Sigma-Aldrich) mixture as the dispersed 80 (droplet) phase and silicon oil (85409, Sigma-Aldrich) as the continuous phase was employed, in order to match the refractive indices of both fluids and the PDMS to avoid optical distortion ${ }^{13}$ due to the droplet curvature. This has also been done in the majority of the reported $\mu \mathrm{PIV}$ investigations. However, in many 85 practical applications other fluid systems are utilised. Thus, in the present study a total of four different droplet-carrier fluid mixtures, notated $\mathrm{S}_{1}, \mathrm{~S}_{2}, \mathrm{~S}_{3}, \mathrm{~S}_{4}$, were investigated in order to examine the effects of viscosity ratio and surfactants; the properties of these systems are summarised in Table 1. The 90 surfactant loaded in the oil phase HFE7500 (297730-93-9, 3M, $0.77 \mathrm{cSt}$ ), Pico-Surf ${ }^{\mathrm{TM}} 1$ (PS-1), was supplied by Sphere Fluidics Ltd (UK). The dynamic viscosity values of water and water/glycerol mixture were obtained from the literature, ${ }^{23,24}$ whereas those of HFE 7500 and silicon oil were obtained from 95 supplier product information sheets. The low concentration of PS-1 in HFE 7500 was considered not to alter the oil viscosity.

The interfacial tension of the working fluids was measured using the pendant droplet method, ${ }^{25}$ which is an established technique. Measurements were performed at room temperature on a 100 FTA1000 B Class contact angle instrument (First Ten Ångstroms, USA) equipped with a camera for video recording. 
Table 1 Dynamic viscosity $(\mu)$, inner-to-outer viscosity ratio $(\lambda)$, and interfacial tension $(\gamma)$, of each fluid for the four droplet-carrier fluid combinations studied.

\begin{tabular}{|c|c|c|c|c|c|c|}
\hline System & Droplet phase & $\begin{array}{c}\mu_{d} \\
(\mathrm{mPa} \cdot \mathrm{s})\end{array}$ & Continuous Phase & $\begin{array}{c}\mu_{c} \\
(\mathrm{mPa} \cdot \mathrm{s})\end{array}$ & $\begin{array}{c}\gamma \\
(\mathrm{mN} / \mathrm{m})\end{array}$ & $\lambda$ \\
\hline $\mathrm{S}_{1}$ & Water/glycerol & 11.5 & Silicon oil & 100 & 30.0 & 0.12 \\
\hline $\mathrm{S}_{2}$ & Water & 1.00 & HFE7500 + PS-1 & 1.28 & 5.2 & 0.78 \\
\hline $\mathrm{S}_{3}$ & Water/glycerol & 11.5 & HFE7500 & 1.28 & 20.2 & 8.98 \\
\hline $\mathrm{S}_{4}$ & Water/glycerol & 11.5 & HFE7500 + PS-1 & 1.28 & 4.0 & 8.98 \\
\hline
\end{tabular}

\section{MicroPIV set up and image acquisition}

(a)
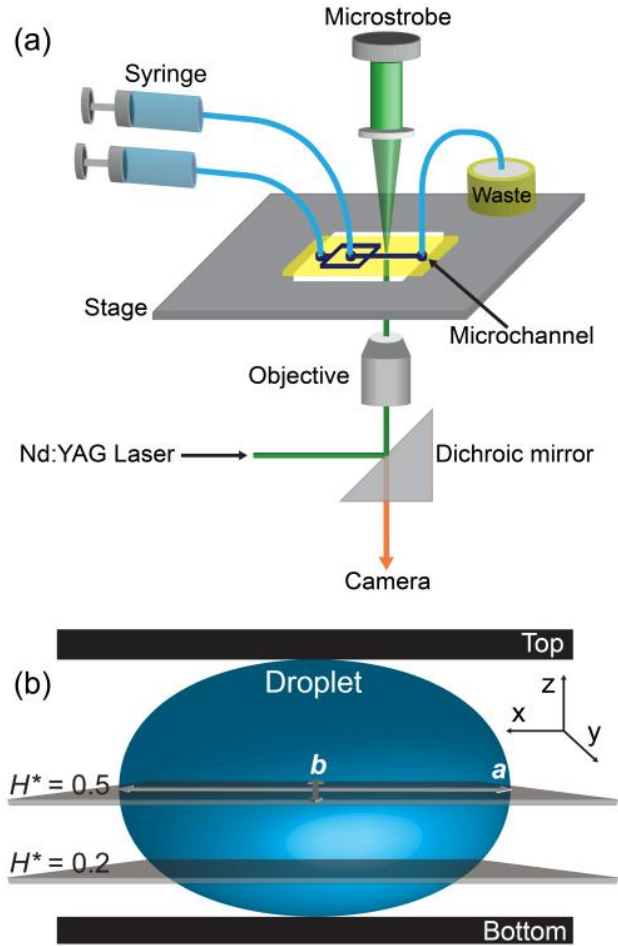

(c) $\mathrm{S}_{1}$ droplet
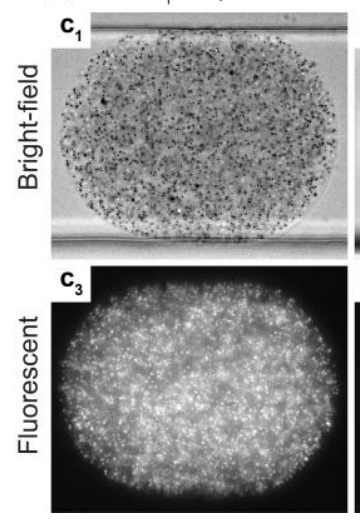

$\mathrm{S}_{2}$ droplet

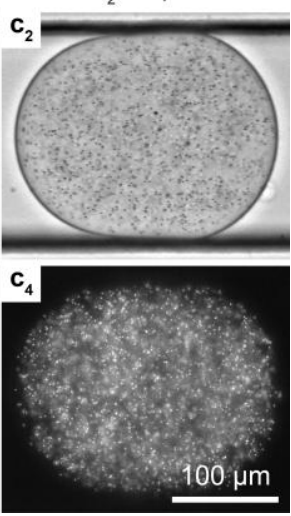

Fig. 2 Image acquisition system. (a) Schematic of the $\mu$ PIV setup used in the present study showing the microchannel and flow delivery system, the light sources, the microscope unit and the image recording system, i.e. the camera. (b) Illustration of the focal 10 planes across the droplet where images were acquired (i.e. $H^{*}=0.2$ and 0.5 , respectively). The droplet dimensions in $x, y$ directions are notated $a$ and $b$, respectively, and the aspect ratio $\beta=a / b$. (c) Typical bright-field ( $\left.\mathrm{C}_{1}, \mathrm{C}_{2}\right)$ and fluorescent $\left(\mathrm{c}_{3}, \mathrm{C}_{4}\right)$ droplet images of two different fluid systems explored in this paper: $S_{1}\left(\mathrm{C}_{1}, \mathrm{C}_{3}\right)$ and $\mathrm{S}_{2}$ 15 (c2, c4) droplets.

The experiments were conducted using a $\mu$ PIV system developed by Sherwood et al. ${ }^{26}$ for studies of microscale blood flow. The system makes use of alternate strobe and laser illumination and is amenable to imaging two phase flows, particularly suspensions. 20 A schematic of the system is shown in Fig. 2a. The microfluidic device was mounted onto the stage of an inverted microscope (Leica DM ILM, Germany). The two fluids were pumped into the microchannel from suitably sized syringes at various flow rates. The aqueous phase was seeded with Nile red fluorescent particles 25 as described in the previous section and excited by illuminating them using a dual cavity Nd:YAG laser (532 nm) (New Wave, USA); the laser light was delivered to the microscope via a liquid light guide. The emitted fluorescence light passed through a dichroic mirror/filter cube with a wavelength of $610 \mathrm{~nm}$ and a 30 bandwidth of $75 \mathrm{~nm}$. Microstrobe illumination was used alternately to observe droplet formation through the objective lens, and enable positioning of the focal plane and monitoring of the morphology and motion of the droplets.

Both fluorescent and strobe images were acquired with a 35 Hamamatsu C8484-05CP CCD (Hamamatsu, Japan) camera in double-frame mode at $6 \mathrm{~Hz}$ through a NA $=0.40,20 \times$ objective lens, resulting in a depth of correlation of $14.8 \mu \mathrm{m}$ based on the formula derived by Olsen and Adrian. ${ }^{27}$ Triggering and image acquisition were carried out using Labview (National

${ }_{40}$ Instruments, USA). The focal plane was varied by adjusting the height of the microscope objective. Fig. $2 b$ illustrates the two focal planes where images were acquired. The normalised vertical plane location is defined as $H^{*}=H / d$, where $H$ and $d$ represent the vertical location of the objective plane relative to the bottom 45 of the channel and the channel depth, respectively. The droplet dimensions in the $x, y$ axes are termed $a$ and $b$ respectively, and 
the aspect ratio is defined as $\beta=a / b$. Fig. $2 \mathrm{c}$ shows typical brightfield and corresponding fluorescent images for two of the fluid systems investigated, one with refractive index matching $\left(S_{1}\right)$ and one without $\left(\mathrm{S}_{2}\right)$.

\section{${ }_{5}$ Data processing}

$\mu$ PIV measurements are Eulerian by nature and in order to crosscorrelate the acquired images and obtain the droplet flow field in the reference frame of the moving droplet (Lagrangian frame) the convective velocity of the droplets needs be subtracted. Henkel et $10 \mathrm{al}^{12}$ solved this issue by operating a droplet matching algorithm (bilinear interpolation of two frames) before PIV analysis. In the present study, a simple processing algorithm was carried out in Labview. Each image pair was first processed to ensure that it contained a full droplet. The images were binarised, and 15 repeatedly dilated so that the droplet could be analysed as a single particle. The image was accepted if the borders of the droplet were not in contact with the image edges. The centroids of the binarised images were then used to locate the centre of the droplet. Both images in each pair were then shifted so that the 20 centroid was geometrically central in the image, thereby subtracting the convective velocity of the droplet. The distance between the two centroids divided by the time between images yielded the droplet velocity. For each case, 100 image pairs were acquired, from which 30 - 50 image pairs were selected and pre-

25 processed using the above algorithm. The coefficient of variation of the droplet velocities was $2 \% \pm 4 \%$.

The time between image pairs was adjusted for each flow rate. After pre-processing, the captured image pairs were crosscorrelated using the open source software JPIV. Three pass 30 ensemble correlation with a final window size of $32 \times 32$ pixels and a vector spacing of 16 pixels was employed resulting in a velocity vector field with a spatial resolution of $6.4 \mu \mathrm{m}$. The monodispersity of the droplets generated in the microfluidic device ensured that the ensemble correlation ${ }^{11,28,29}$ of $30-50$ 35 image pairs was sufficient to minimise outliers. The normalised median test ${ }^{30}$ was applied to identify outliers, which frequently appeared at the edges of the data field; the invalid vectors were replaced by the median of the surrounding vectors. Finally, the vector field was smoothed with a $3 \times 3$ median filter. ${ }^{31}$

\section{${ }_{40}$ Effect of optical distortion}

The refractive index mismatch between the two liquid phases is likely to introduce some errors due to optical distortion caused by the curved interface. However, matching the refractive index in droplet systems of practical interest is a challenging task. In the 45 present study, refractive index matching was achieved in system $\mathrm{S}_{1}$, similarly to Kinoshita et al. ${ }^{13}$ and hence this system can be considered to be the benchmark case for the $\mu$ PIV measurements. However, the refractive index of water at $20^{\circ} \mathrm{C}$ is 1.33 , while that of all commercial fluorinated oil like HFE7500, FC40, FC3283, 50 etc. is below 1.30. Hence, the refractive indices of aqueous and fluorinated oil phases cannot be perfectly matched in all measured systems. In order to assess the degree of uncertainty introduced by the mismatch, a ray tracing methodology ${ }^{32}$ was implemented in Matlab (Mathworks, USA) to study the 55 distortion. The distortion in the water droplet in HFE7500 $\left(\mathrm{S}_{2}\right)$ was found to be negligible for plugs. The distortion in the water/glycerol (45/55, v/v) mixture droplet in $\operatorname{HFE7500~}\left(\mathrm{S}_{3}\right.$ and
$\mathrm{S}_{4}$ ) was found to be more significant than that in $S_{2}$, but for plugs was confined to a small region in the droplet caps and should not 60 affect the general flow pattern observed therein. Thus it can be concluded that in the present study, the errors due to optical distortion can be neglected and the measured quantities are reasonably accurate.

\section{Results and Discussion}

\section{${ }_{65}$ Droplet mobility}
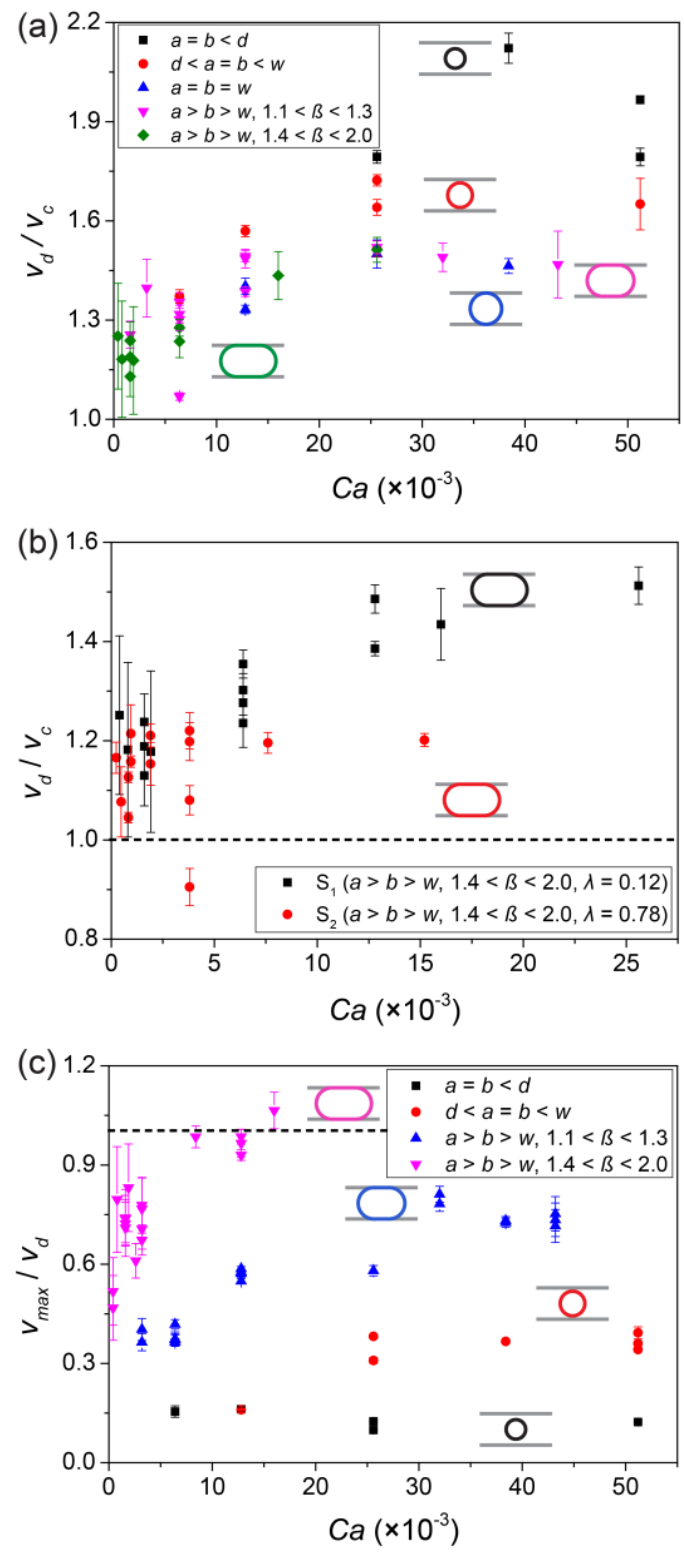

Fig. 3 (a) Droplet mobility ratio, $v_{d} / v_{c}$, as a function of $\mathrm{Ca}$ and droplet geometry for $S_{1}(\lambda=0.12)$ droplets and $(b)$ comparison of droplet mobility for large plugs of different viscosity ratios, $\mathrm{S}_{1}(\lambda=$ $70 \quad 0.12)$, and $S_{2}(\lambda=0.78) . a=b<d$ represents spherical droplets with no contact with either channel sides; $d<a=b<w$ represents pancake droplets in contact with the channel top and bottom, but not with the side walls; $a=b=w$ represents pancakes droplets touching the side walls but have no significant friction with the top 75 and bottom; $a>b=w$ represents either small or large plugs with different aspect ratios, $\beta$. Error bars show $95 \%$ confidence intervals 
on the mean. Error in $v_{c}$ is considered to be negligible. (c) Maximum velocity in droplet bulk volume, $v_{\max }$, normalised by droplet velocity, $v_{\max } / v_{d}$, plotted against $\mathrm{Ca}$ for four different sized $\mathrm{S}_{1}$ droplets. Uncertainty in the ratio $v_{\max } / v_{d}$ is given by the chain

5 rule method of Kline and McClintock ${ }^{33}$ and illustrated as $95 \%$ confidence intervals. The error in $v_{\max }$ is considered to be negligible.

Figure 3 shows the ratio of droplet velocity, $v_{d}$, to the velocity of the continuous phase, $v_{c}$, as a function of the $C a$ and the aspect ratio of the droplets. In the present study $\mathrm{Ca}$ is defined based on $10 v_{c}$, as $v_{d}$ deviated from the average bulk velocity, $v_{c}$, due to contact with the channel walls; hence $C a=\mu_{c} v_{c} / \gamma$, where $\mu_{c}$ is the viscosity of the continuous phase, and $\gamma$ is the interfacial tension. Figure 3 shows that the mobility of the droplets is affected by $\mathrm{Ca}$, droplet geometry (Fig. 3a) and inner-to-outer viscosity ratio, $\lambda$ 15 (Fig. 3b). At low viscosity ratio (Fig. 3a) the droplet velocity is higher than that of the continuous phase for the $C a$ range and droplet shapes studied. The $v_{d} / v_{c}$ ratio is higher for spherical droplets, as expected due to the absence of wall effects and the intrinsic parabola-like flow profile in microchannels. ${ }^{34}$ On the 20 contrary, plugs $(a>b=w)$ experience increased contact with the channel walls, allowing the continuous phase to bypass them only through the corners; a long thin film layer is formed between the channel walls and the droplet exerting substantial shear forces (drag) on the droplet and causing its velocity $v_{d}$ to reduce 25 compared to that of spherical droplets and to become closer to $v_{c}$. The mobility of pancake droplets $(d<a=b<w)$ lies between those of spherical droplets and plugs due to contact with two of the channel walls. Within the studied $C a$ range, the velocity ratio is always greater than unity, but the rate of increase in mobility 30 with increasing $\mathrm{Ca}$ in plugs is smaller compared to spherical droplets and pancakes.

Trends were analysed using Spearman's rank correlation coefficient to account for the non-linear relationships observed in Fig. 3. Means were evaluated using the Mann-Whitney U test. In ${ }_{35}$ Fig. 3a, the trend in increasing $v_{d} / v_{c}$ with increasing $\mathrm{Ca}$ was moderately significant for $a<w$ (black and red), giving $r=$ $0.6779, p=0.0312$. For the plug droplets $a>w$ (green and pink), a highly significant correlation was observed: $r=0.7928, p<$ 0.0001 .

${ }_{40}$ Jakiela et al. ${ }^{20}$ observed a discontinuous transition in the mobility of droplets as a function of $\mathrm{Ca}$ which was attributed to a transition in flow topology. However, the aspect ratio of the droplets they studied was higher, and the $C a$ lower, than in the present work; hence no such transition could be observed in the 45 present study.

Figure $3 b$ illustrates the effect of viscosity ratio $\lambda$ on droplet mobility. For low $\mathrm{Ca}(<2.5)$ there was no significant difference in $v_{d} / v_{c}$ between $\mathrm{S}_{1}$ and $\mathrm{S}_{2}$ droplets $(p=0.108)$. However, as $\mathrm{Ca}$ increased, $v_{d} / v_{c}$ for $\mathrm{S}_{1}$ droplets increased $(r=0.8113, p=0.0004)$ 50 while for $\mathrm{S}_{2}$ droplets it did not $(r=0.3189, p=0.2665)$.

The effect of $\mathrm{Ca}$ on the internal maximum velocity in the droplet bulk volume is shown in Fig. $3 c$ for $S_{1}$ droplets exhibiting the typical circulation pattern reported in the literature. ${ }^{13}$ The maximum velocity, $v_{\max }$, is obtained from the ensemble averaged 55 velocity data measured in the central focal plane, and normalised with the droplet velocity $v_{d}$. The $v_{\max } / v_{d}$ relationship is strongly dependent on the droplet geometry. The relative maximum velocity for spherical and pancake droplets $(a<w, p>0.15)$ is independent of $\mathrm{Ca}$ and is much lower than the droplet velocity. ${ }_{60}$ However, as the droplet aspect ratio, $\beta$, increases, $C a$ has a noticeable effect for both $1.1<\beta<1.3(r=0.8895, p<0.0001)$ and $1.4<\beta<2.0$ ( $r=0.7131, p=0.0009)$, which appears to increase with aspect ratio. For droplets with aspect ratios greater than $1.4 v_{\max }$ exceeds $v_{d}$ for higher $\mathrm{Ca}$. As the droplet length 65 increases, the surface area in contact with the microchannel walls increases. The droplet/channel friction, or more precisely, the shear stresses across the w/o interface, induce the internal flow circulation, and higher shear stresses thus lead to larger $v_{\max } / v_{d}$ in the plugs.

\section{${ }_{70}$ Droplet velocity field}

Figure 4 illustrates the measured velocity characteristics of $S_{1}$ droplets with a low viscosity ratio of 0.12 . All velocities are presented in the reference frame of the droplet with positive velocities indicating flow in the direction of the droplet. Typical 75 vector fields are shown in Figs. $4 \mathrm{a}$ and $4 \mathrm{~b}$ representing the flow topologies in the central focal planes $\left(H^{*}=0.5\right)$ of two different sized droplets: a large plug at $C a=6.4 \times 10^{-3}$ (a) and a spherical droplet at $C a=1.0 \times 10^{-1}$ (b). For clarity of presentation, every other vector is omitted. The flow topology is similar in both 80 vector plots: the central region of the droplet exhibits parabolalike flow in the same direction as the droplet motion, surrounded by two counter rotating recirculation zones on either side of the droplet axis. Figures $4 \mathrm{c}$ to $4 \mathrm{f}$ show the contours of the two velocity components. The axial and transverse velocities are 85 normalised by the droplet velocity (i.e. $\bar{u} / v_{d}$ and $\bar{v} / v_{d}$ ). Qualitatively, the distributions of the two velocity components are similar between the plug and the spherical droplet; however the velocity magnitudes differ, with the plugs exhibiting higher $\bar{u} / v_{d}$ and $\bar{v} / v_{d}$ than spherical droplets (note different colour scales 90 in Fig. 4). It was found that this topology persisted for $\mathrm{Ca}$ ranging from $10^{-3}$ to $10^{-1}$, and droplet geometries ranging from large plugs $(a / b=2.0)$ to spherical droplets $(a=b<d)$. 


\section{Cite this: DOI: $10.1039 / \mathrm{c0xx00000x}$}
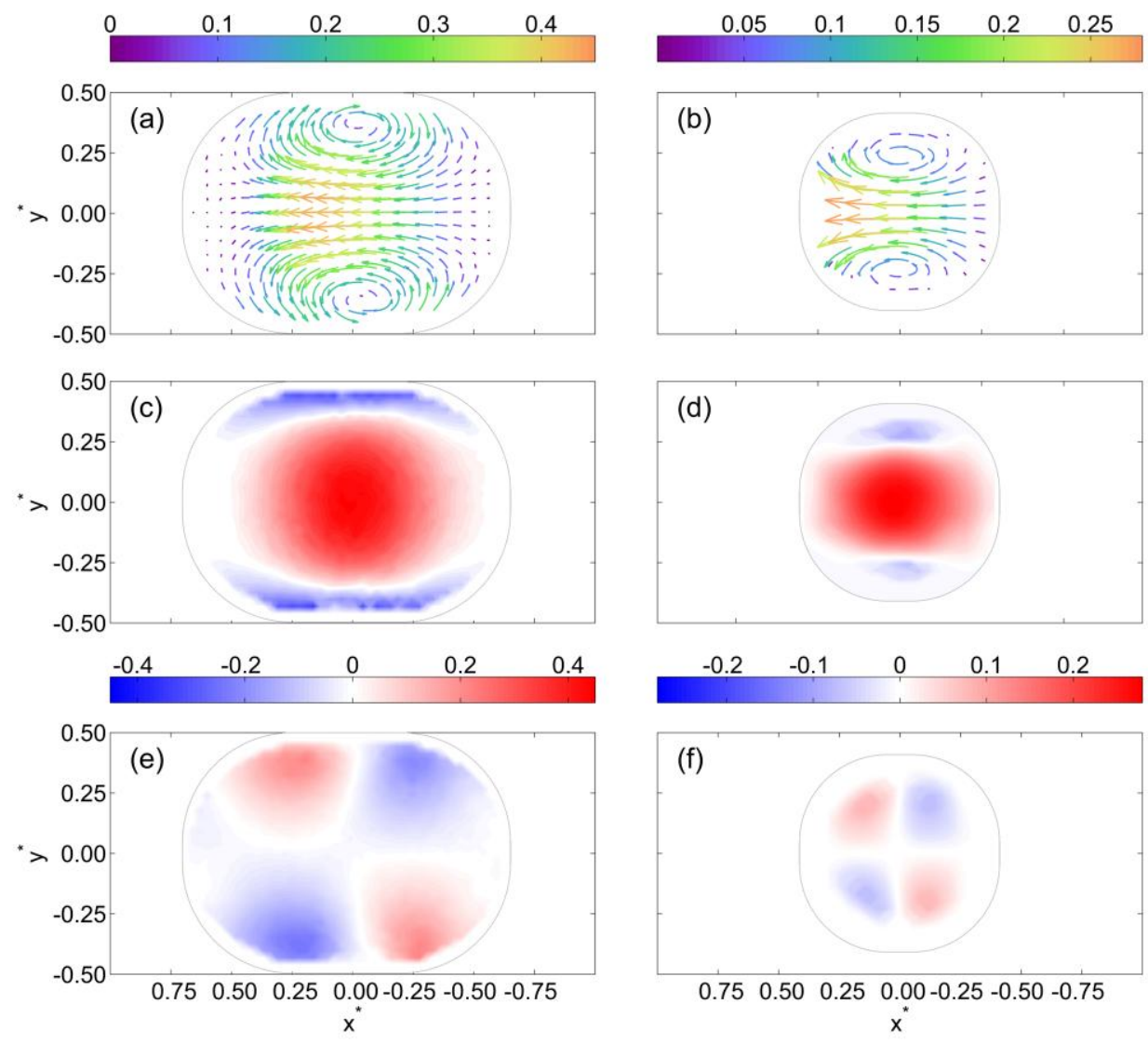

Fig. $4(a, b)$ Velocity vector fields - every other vector is omitted for clarity of presentation, $(c, d)$ axial velocity component contours and (e, $f)$ transverse velocity component contours at the mid-plane $\left(H^{*}=0.5\right)$. $(\mathrm{a}, \mathrm{c}, \mathrm{e})$ show a large $\mathrm{S}_{1}$ plug: $\mathrm{Ca}=6.4 \times 10^{-3}, v_{d}=2.2 \mathrm{~mm} / \mathrm{s}$. $(\mathrm{b}, \mathrm{d}, \mathrm{f}) \mathrm{show}$ 5 a spherical $S_{1}$ droplet: $C a=1.0 \times 10^{-1}, v_{d}=57.7 \mathrm{~mm} / \mathrm{s}$. Velocity components $\left(\bar{u} / v_{d}\right.$ and $\left.\bar{v} / v_{d}\right)$ are normalised by droplet velocity, $v_{d}$, and spatial co-ordinates $\left(x^{*}, y^{*}\right)$ are normalised by the channel width, $w$, and defined relative to the centre of the droplet.

\section{Effect of viscosity ratio on flow topology}

It is clear from the above that the droplet geometry and $\mathrm{Ca}$ do not affect the flow topology, but only the magnitude of flow 10 velocities. However, a change in flow topology was observed in the present study for certain fluid mixtures and it is interesting to probe the physical factors that caused the change. To indicate the topology change more clearly the velocity vectors measured at two focal planes in the two fluid systems are compared in Figs. 5 15 and 6. Fig. 5 shows the vector plots of a large $S_{1}$ plug at $H^{*}=0.2$ (a) and $H^{*}=0.5$ (b), and the corresponding contours of the normalised axial and transverse velocity components, $\bar{u} / v_{d}$ (c, d) and $\bar{v} / v_{d}(\mathrm{e}, \mathrm{f})$, respectively. The lower plane $\left(H^{*}=0.2\right)$ of the $S_{1}$ plug has a flow topology with opposite directionality to that in
20 the central plane $\left(H^{*}=0.5\right)$ and with both velocity components exhibiting lower magnitude. The aspect ratio of the channel is $4: 3$, and it is reasonable to assume symmetry in the $Y Z$ plane, i.e. the droplet central region will exhibit maximum axial velocities, accompanied by recirculating flows close to channel boundaries.

${ }_{25}$ Figs. $6 \mathrm{a}$ and $6 \mathrm{~b}$ show vector plots of a water droplet $\left(\mathrm{S}_{2}\right)$ moving in HFE7500, loaded with $1.5 \mathrm{wt} \%$ PS-1 surfactant (to promote droplet formation and stabilization). Unlike the topology observed for the $\mathrm{S}_{1}$ droplet, the central plane of the $\mathrm{S}_{2}$ droplet $\left(H^{*}\right.$ $=0.5$, Figs. $6 \mathrm{~b}, \mathrm{~d}$ ) exhibits low magnitude, near zero, velocities in 30 the central region, accompanied by two regions of relatively higher magnitude negative velocities (in a direction opposite to the droplet motion) on either side of the centreline and close to the w/o interface and channel walls. Closer to the bottom wall 
$\left(H^{*}=0.2\right.$, Figs. 6a, c), negative axial velocities are observed in the central droplet region and more pronounced positive velocities are observed on either side of the centreline. The transverse velocities (Figs. 6e, f) exhibit very low values 5 indicating negligible momentum transfer across the droplet centreline.
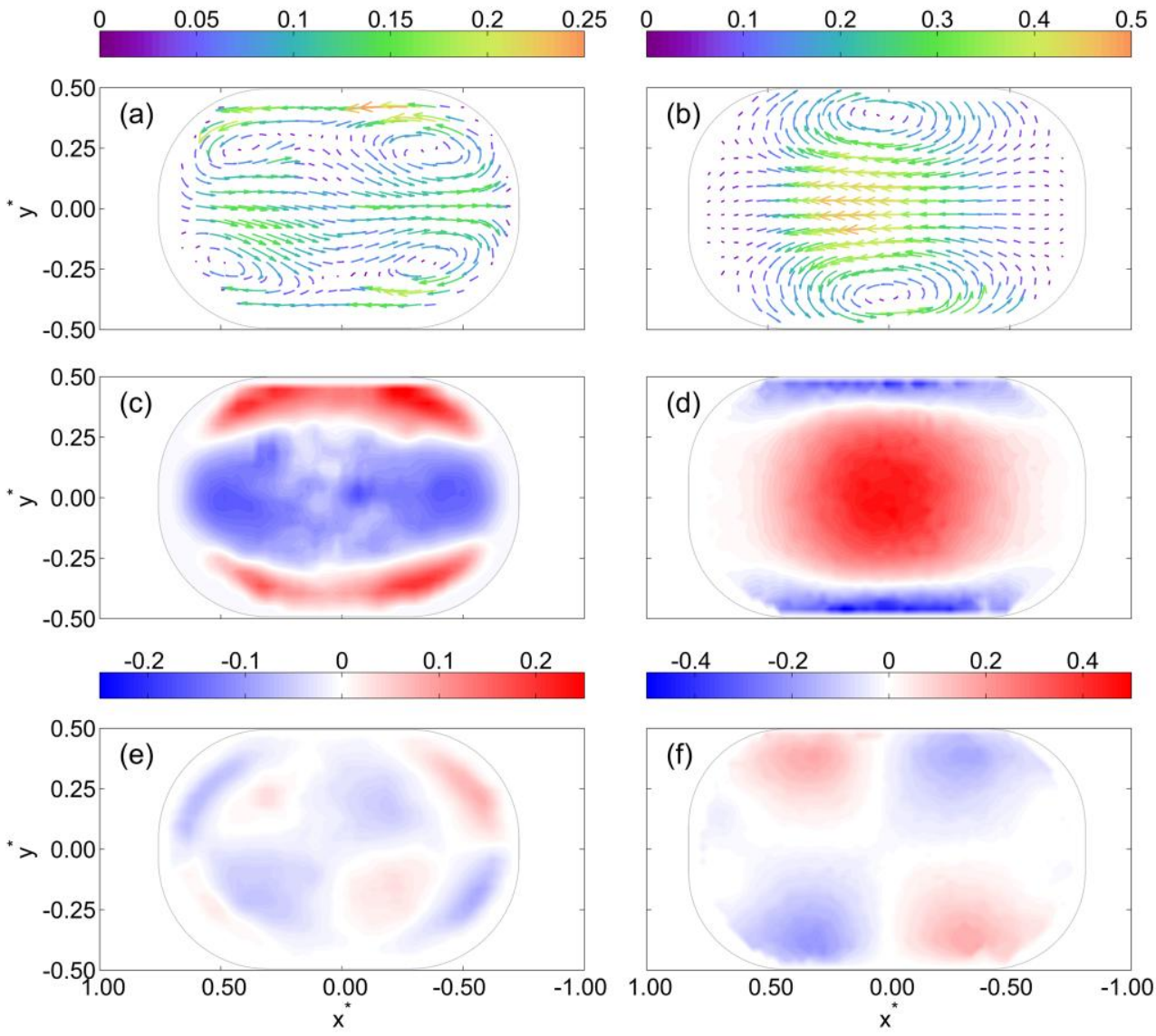

Fig. $5(a, b)$ Velocity vector fields - every other vector is omitted for clarity of presentation, $(c, d)$ axial velocity component contours and (e, f) 10 transverse velocity component contours of a large $S_{1}$ plug $(\lambda=0.12): C a=1.8 \times 10^{-3}, v_{d}=0.6 \mathrm{~mm} / \mathrm{s} .(\mathrm{a}, \mathrm{c}, \mathrm{e})$ show the lower focal plane $\left(H^{*}=\right.$ $0.2)$, and $(\mathrm{b}, \mathrm{d}, \mathrm{f})$ show the mid-plane $\left(H^{*}=0.5\right)$. All velocity components $\left(\bar{u} / v_{d}\right.$ and $\left.\bar{v} / v_{d}\right)$ are normalised by droplet velocity, $v_{d}$, and spatial co-ordinates $\left(x^{*}, y^{*}\right)$ are normalised by the channel width, $w$, and defined relative to the centre of the droplet. It should be noted that the $S_{1}$ plug shown here is longer than that in Fig. 4a, in order to match the length of the $S_{2}$ plug in Fig. 6 for better comparison.

Figures 5 and 6 indicate that the increased viscosity ratio in 15 system $\mathrm{S}_{2}$ alters the circulation dynamics of the droplets. The observed changes in flow topology can be explained by examining the boundary conditions that apply in this flow. At the wall, the no-slip boundary condition holds, whereas at the interface between the continuous and the droplet phase, notated $c$ 20 and $d$, respectively, the velocities tangent to the interface and the corresponding shear stresses should match, i.e.

$$
\left.\mu_{d} \frac{\partial u}{\partial y}\right|_{d}=\left.\mu_{c} \frac{\partial u}{\partial y}\right|_{c}
$$

It should be noted that according to Guillot et al.,${ }^{35}$ the interface shear viscosity term can be neglected when considering the shear 25 stresses at the liquid-liquid interface, as the interface shear viscosity tends to be substantially smaller than the fluid viscosity in the presence of surfactant monolayers at the w/o interface. It is clear that the boundary conditions dictate the droplet flow topology to a large extent and the viscosity ratio $\lambda=$
${ }_{30}(\partial u / \partial y)_{c} /(\partial u / \partial y)_{d}$ plays a key role. Thus to ensure velocity continuity at the w/o interface, the axial velocities in the central plane, near wall region of both $S_{1}$ and $S_{2}$ droplets (Figs. $5 \mathrm{~d}$ and 6d) become negative and equal in magnitude to those of the thin film layer that exists between the w/o interface and the channel 35 wall. In the reference frame of the droplet, both the channel wall and the thin film layer will exhibit negative velocities, i.e. will flow in a direction opposite to the droplet motion, due to the noslip boundary condition at the wall. At the same time, the viscosity ratio of 0.12 in $S_{1}$ droplets implies that the velocity 40 gradient $(\partial u / \partial y)_{d}$ at the w/o interface inside the droplet should be around 8 times larger than the corresponding gradient in the thin film layer $(\partial u / \partial y)_{c}$ to satisfy Equation (1), which results in flow reversal in the interior of the droplet and establishment of the well-known recirculating pattern of Fig. 5b. In contrast, the 45 viscosity ratio in $S_{2}$ droplets is 0.78 , i.e. close to 1 , which implies that the velocity gradients, inside and outside the droplet are comparable. Hence the (net) droplet internal velocities remain low and flow does not reverse in the droplet interior. The droplet 
circulation pattern is no longer apparent.
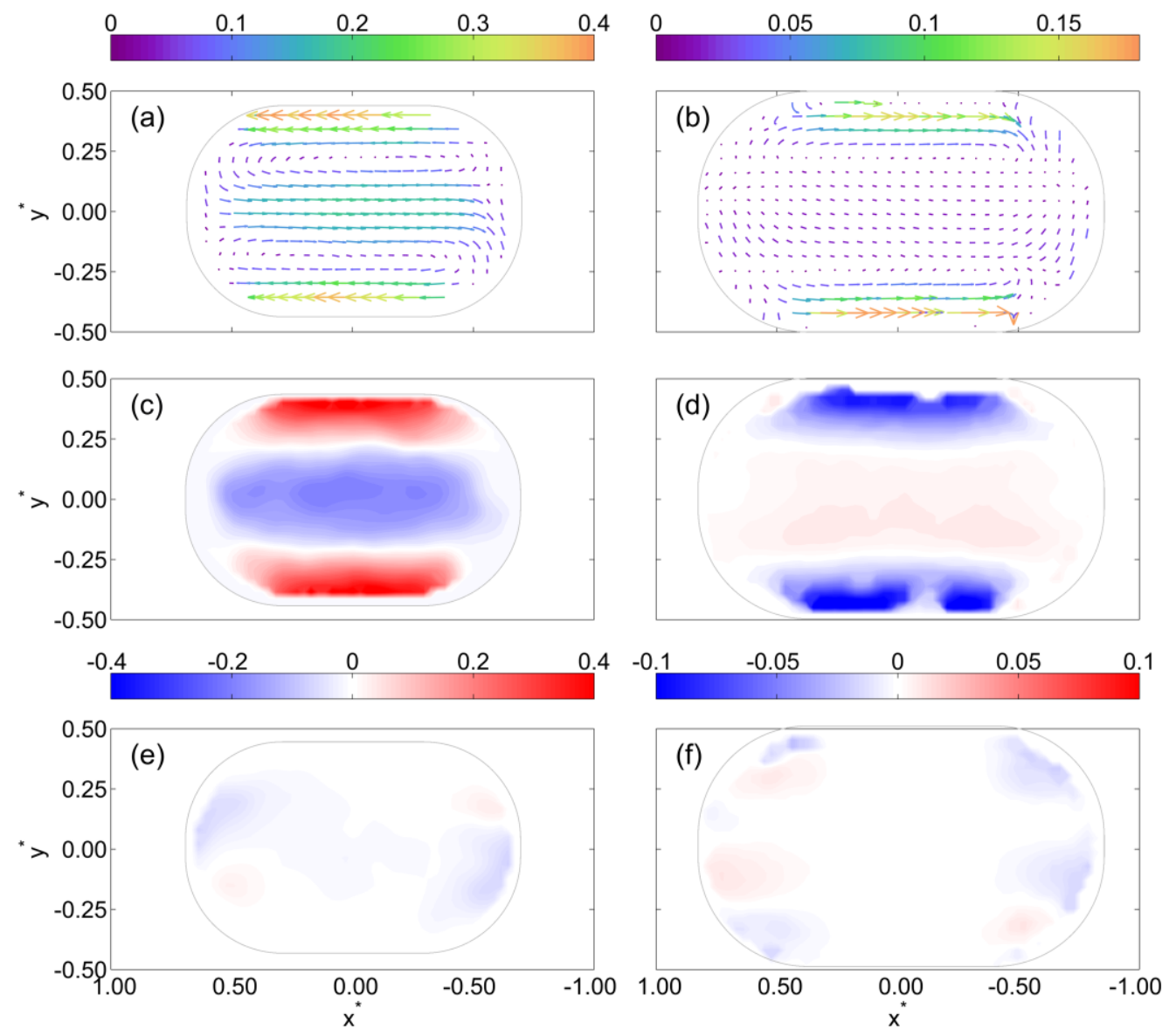

Fig. $6(a, b)$ Velocity vector fields - every other vector is omitted for clarity of presentation, $(c, d)$ axial velocity component contours and (e, f) transverse velocity component contours of a large $S_{2}$ plug $(\lambda=0.78): C a=3.8 \times 10^{-3}, v_{d}=16.0 \mathrm{~mm} / \mathrm{s}$. $\left(\mathrm{a}, \mathrm{c}\right.$, e) show the lower focal plane $\left(H^{*}=\right.$ $50.2)$, and $(\mathrm{b}, \mathrm{d}, \mathrm{f})$ show the mid-plane $\left(H^{*}=0.5\right)$. All velocity components $\left(\bar{u} / v_{d}\right.$ and $\left.\bar{v} / v_{d}\right)$ are normalised by droplet velocity, $v_{d}$, and spatial co-ordinates $\left(x^{*}, y^{*}\right)$ are normalised by the channel width, $w$, and defined relative to the centre of the droplet.

The distribution of axial velocities in the lower focal planes (Figs. $5 \mathrm{c}$ and $6 \mathrm{c}, H^{*}=0.2$ ) shows positive axial velocities along the w/o interface. The difference between this flow topology and that 10 observed at $H^{*}=0.5$ can be attributed to the 'gutter' flow of the continuous phase in the rectangular channel, a factor that differentiates this flow from the extensively studied droplet flow in circular capillaries. When a droplet flows in a rectangular channel, the corners of the channel are not invaded by the droplet 15 but filled with the continuous fluid. Wong et al. ${ }^{36}$ described the flow of long bubbles through polygonal capillaries as leaky pistons in which the flow either pushes the bubble (plug flow) or bypasses it through the corners (corner flow) and postulated that the corner flow is unidirectional due to the length of the bubbles.

${ }_{20}$ Garstecki et al. ${ }^{20}$ observed that the corner or gutter flow was always oriented forward, i.e. in the same direction as the moving droplet. This explains the positive axial velocities near the interface in all contours at $H^{*}=0.2$, since the velocities at the interface between the two phases should match. The negative 25 velocities measured in the central region of this focal plane can be attributed to the vicinity of the bottom channel wall and the thin film layer therein.

\section{The role of interfacial tension}

The two systems, $S_{1}$ and $S_{2}$, examined above differ not only in 30 the inner-to-outer viscosity ratio, $\lambda$, but also in interfacial tension, $\gamma$, as $\mathbf{S}_{2}$ contains a small quantity of surfactant (see Table 1 ). It is well known that the existence of surfactant absorbed on the w/o interface induces Marangoni stresses, which retard the boundary flow in a manner analogous to increasing the viscosity of the 35 droplet fluid. ${ }^{37}$ It is therefore prudent to examine whether the interfacial tension and Marangoni effects played a role in the flow topology change observed in the previous section. This was achieved by performing additional flow measurements with droplet systems in which the interfacial tension $\gamma$ was varied and $\lambda$ 40 was kept constant. The results are shown in Fig. 7.

Two additional fluid systems were tested: $S_{3}$, comprising water/glycerol mixture (45/55, v/v) droplets in HFE7500, without surfactant (Figs. 7a, c), and $\mathrm{S}_{4}$, with the same composition but with HFE7500 loaded with 1.5 wt\% PS-1 (Figs. 7b, d). The 45 viscosity ratio is 8.98 for both systems, which is substantially higher than those of systems $\mathbf{S}_{1}$ and $\mathbf{S}_{2}$. The difference in $\gamma$ between $S_{1}$ with $S_{3}$ is relatively small $\left(\gamma_{3} / \gamma_{1}=2 / 3\right)$, compared to 
that between $S_{1}$ and $S_{2}\left(\gamma_{2} / \gamma_{1}=1 / 6\right)$. A comparison between the droplet flow patterns in $S_{1}$ and $S_{3}$ (Figs. 5a, b and Figs. 7a, c respectively) suggests that the change in topology from $S_{1}(\lambda<1)$ to $S_{3}(\lambda>1)$ is attributable to the increase in $\lambda$. Droplet circulation 5 is suppressed and a more uniform flow pattern is established in the more viscous $\mathrm{S}_{3}$ droplets which can be explained on the basis of Equation (1). The observed changes in the droplet flow pattern are also illustrated analytically by Jin et $a l .{ }^{38}$ for capillary droplet flows. Their analytical velocity profiles showed that at low $\lambda$, the 10 droplet tended to adopt a parabola-like topology in the bulkvolume, whereas with increasing $\lambda$, a more uniform distribution was established. However, such analytical solutions are not valid for droplet flows in rectangular channels and should be treated with caution.

${ }_{15} \mathrm{~S}_{3}$ and $\mathrm{S}_{4}$ droplets exhibit qualitatively similar flow topologies (Figs. 7a, c and 7b, d) despite a factor of 5 difference in interfacial tension. This suggests that Marangoni effects and w/o interfacial tension do not play an important role in the altered droplet flow dynamics observed in the present study. According 20 to Griffiths et al. ${ }^{39}$ the added surfactant builds up on the interface of a moving droplet in an asymmetric profile, with an excess of surfactant at the rear of the droplet, in which case, Marangoni effects should induce positive axial velocities along the interface. However, in Fig. $6 \mathrm{~b}\left(H^{*}=0.5\right)$, the axial velocity is negative 25 along the interface, implying dominance of viscous stresses relative to Marangoni stresses. As PS-1 was only loaded at very low concentration (but sufficiently high to reduce $\gamma$ significantly and induce possible Marangoni flow), the viscosity of the oil phase is considered to remain the same in both $S_{3}$ and $S_{4}$. The 30 difference in $\gamma$ between $S_{3}$ and $S_{4}$ is approximately of the same magnitude as between $S_{1}$ and $S_{2}$ (see Table 1), thus the similar flow topologies, observed in these additional fluid systems, exclude the role of $\gamma$ in inducing substantial flow topology changes. It could be that the convective nature of the flow, 35 together with the low surfactant concentration suppresses Marangoni stresses in the present study. For all the systems studied, the flow topology in the inner region of the central focal plane $\left(H^{*}=0.5\right)$ was opposite to that in the central zone of the lower plane $\left(H^{*}=0.2\right)$, due to mass conservation and 40 incompressibility of fluids. ${ }^{13,40,41}$
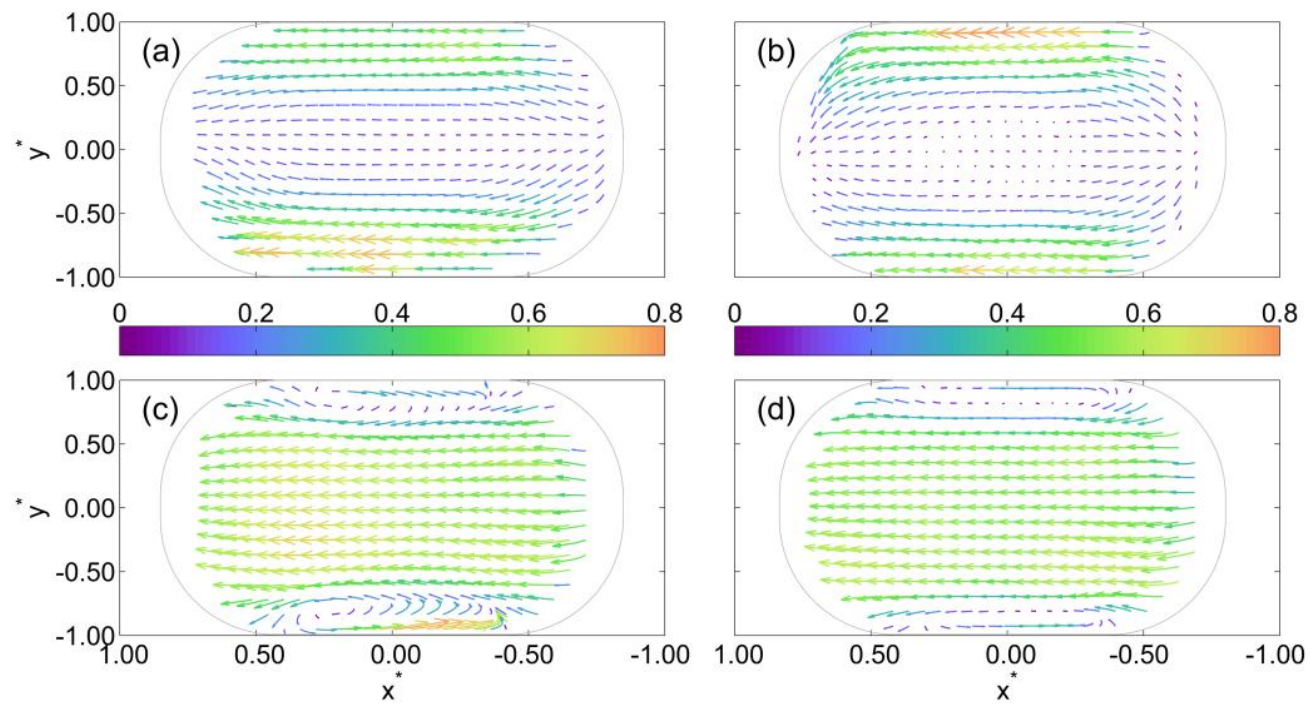

Fig. 7 Velocity vector fields of $(\mathrm{a}, \mathrm{c})$ a large $\mathrm{S}_{3}$ plug $\left(C a=0.46 \times 10^{-3}, v_{d}=8.0 \mathrm{~mm} / \mathrm{s}\right)$, and $(\mathrm{b}, \mathrm{d})$ a large $\mathrm{S}_{4} \mathrm{plug}\left(\mathrm{Ca}=2.3 \times 10^{-3}, v_{d}=8.0 \mathrm{~mm} / \mathrm{s}\right) .(\mathrm{a}$, b) show the lower focal plane $\left(H^{*}=0.2\right)$ and $(c, d)$ show the mid-plane $\left(H^{*}=0.5\right)$. Velocities are normalised by droplet velocity, $v d$, and spatial co-ordinates $\left(x^{*}, y^{*}\right)$ are normalised by the channel width, $w$, and defined relative to the centre of the droplet. Every other vector is omitted for clarity of presentation.

The measured flow fields demonstrate the key role of the viscosity ratio between the two phases in determining droplet flow topology in rectangular microchannels. Although $\mathrm{Ca}$, droplet geometry and viscosity ratio all seem to affect droplet 50 mobility, the internal flow topology is found to be primarily determined by the viscosity ratio of the two phases even in the presence of surfactant in the range of $\mathrm{Ca}$ studied here.

\section{Conclusion}

The droplet interior flow field was studied by means of $\mu$ PIV and 55 a significant flow topology change was observed in droplets moving in a rectangular microchannel at moderate $\mathrm{Ca}, 10^{-3}$ to $10^{-}$
${ }^{1}$. The study shows that the dominant factor inducing the topology change is the viscosity ratio across the w/o interface of the droplet. With $\lambda$ increasing from 0.12 to 0.78 , the droplet 60 undergoes a flow topology change from the high velocity parabola-like profile in the central zone, accompanied by two counter recirculating patterns on each side, to low velocity flow in most of the central zone with high reversing flows confined only along the interface.

${ }_{65}$ Surfactant-induced Marangoni effects were not a dominant factor in the observed flow topology change. $\mathrm{Ca}$ and droplet geometry were found to affect the internal velocity magnitude, and the sizes of the recirculation, but not the intrinsic flow topology. The results shown here indicate that by tuning the viscosity ratio, the 
strength of the internal circulatory motions can be adjusted or even eliminated. A droplet system with higher inner-to-outer viscosity ratio should be adopted when low internal circulations are desired, such as in cell screening applications; however, lower 5 viscosity ratio systems are preferred when aiming for efficient mixing.

\section{Acknowledgement}

SM and WTSH would like to thank Dr V. van Steijn and Dr D. A. Hoang at Delft University of Technology, The Netherlands for 10 useful discussions on $\mu$ PIV diagnostics prior to this study. SM acknowledges the financial support provided by the CSC Cambridge Scholarship throughout his PhD study.

SB would like to thank Dantec Dynamics for the loan of the laser used in this study. Finally the authors wish to thank Dr Peter 15 Vennemann for providing the free PIV analysis software JPIV (http://www.jpiv.vennemann-online.de/).

\section{Notes and references}

${ }^{a}$ Department of Chemistry, University of Cambridge, Cambridge, CB2 $1 E W, U K$

$20{ }^{b}$ Department of Mechanical Engineering, University College London, Torrington Place, London WC1E 7JE, UK. Email: s.balabani@ucl.ac.uk ${ }^{c}$ Department of Bioengineering, Imperial College London, London SW7 $2 A Z, U K$

${ }^{d}$ Radboud University Nijmegen, Institute for Molecules and Materials,

25 Heyendaalseweg 135, 6525 AJ Nijmegen, The Netherlands. Email:

w.huck@science.ru.nl

$\$$ Present address: Department of Chemistry, University of Oxford,

Oxford, OX1 3TA, UK.

$\dagger$ Electronic Supplementary Information (ESI) available: [movies of image 30 acquiring]. See DOI: 10.1039/b000000x/

1. H. Song, J. D. Tice, and R. F. Ismagilov, Angew. Chemie Int. Ed., 2003, 115, 792-796.

2. A. B. Theberge, F. Courtois, Y. Schaerli, M. Fischlechner, C. Abell, F. Hollfelder, and W. T. S. Huck, Angew. Chemie Int. Ed., 2010, 49, $35 \quad 5846-5868$.

3. H. M. Evans, E. Surenjav, C. Priest, S. Herminghaus, R. Seemann, and T. Pfohl, Lab Chip, 2009, 9, 1933-1941.

4. B. Zheng, L. S. Roach, and R. F. Ismagilov, J. Am. Chem. Soc., 2003, 125, 11170-11171.

40 5. M. Chabert and J.-L. Viovy, Proc. Natl. Acad. Sci. U. S. A., 2008, 105, 3191-3196.

6. T. Yoshioka, R. Hirano, T. Shioya, and M. Kako, Biotechnol. Bioeng., 1990, 35, 66-72.

7. H. Kong, M. K. Smith, and D. J. Mooney, Biomaterials, 2003, 24, 45 4023-4029.

8. E. Brouzes, M. Medkova, N. Savenelli, D. Marran, M. Twardowski, J. B. Hutchison, J. M. Rothberg, D. R. Link, N. Perrimon, and M. L. Samuels, Proc. Natl. Acad. Sci. U. S. A., 2009, 106, 14195-14200.

9. S. Tasoglu, U. A. Gurkan, S. Wang, and U. Demirci, Chem. Soc. Rev., 2013, 42, 5788-5808.

10. R. Lindken, M. Rossi, S. Grosse, and J. Westerweel, Lab Chip, 2009, 9, 2551-2567.

11. S. T. Wereley and C. D. Meinhart, Annu. Rev. Fluid Mech., 2010, 42, 557-576.

55 12. D. Malsch, M. Kielpinski, R. Merthan, J. Albert, G. Mayer, J. M. Köhler, H. Süße, M. Stahl, and T. Henkel, Chem. Eng. J., 2008, 135S, S166-S172.

13. H. Kinoshita, S. Kaneda, T. Fujii, and M. Oshima, Lab Chip, 2007, 7, 338-346.

60 14. L. Prat, C. Gourdon, F. Sarrazin, K. Loubie, T. Bonometti, and J. Magnaudet, AIChE J., 2006, 52, 4061-4070.

15. C. King, E. Walsh, and R. Grimes, Microfluid. Nanofluidics, 2007, 3, 463-472.
16. U. Miessner, R. Lindken, and J. Westerweel, in 14th Int Symp on Applications of Laser Techniques to Fluid Mechanics, 2008, pp. 7 10.

17. V. Dore, D. Tsaoulidis, and P. Angeli, Chem. Eng. Sci., 2012, 80, 334-341.

18. M. Oishi, H. Kinoshita, T. Fujii, and M. Oshima, J. Phys. Conf. Ser., 2009, 147, 012061

19. V. Labrot, M. Schindler, P. Guillot, A. Colin, and M. Joanicot, Biomicrofluidics, 2009, 3, 12804.

20. S. Jakiela, P. M. Korczyk, S. Makulska, O. Cybulski, and P. Garstecki, Phys. Rev. Lett., 2012, 108, 134501.

75 21. Y. Xia and G. M. Whitesides, Аnnu. Rev. Mater. Sci., 1998, 28, $153-$ 184.

22. W.-A. C. Bauer, M. Fischlechner, C. Abell, and W. T. S. Huck, Lab Chip, 2010, 10, 1814-1819.

23. J. Kestin, M. Sokolov, and W. A. Wakeham, J. Phys. Chem. Ref. Data, 1978, 7, 941-948.

24. N. S. Cheng, Ind. Eng. Chem. Res., 2008, 47, 3285-3288.

25. X. Wang, A. Brandvik, V. Alvarado, Energy Fuels, 2010, 24, 63596365.

26. J. M. Sherwood, E. Kaliviotis, J. Dusting, and S. Balabani, Biomech. 85 Model. Mechanobiol., 2014, 13, 259-273.

27. M. G. Olsen and R. J. Adrian, Exp. Fluids, 2000, 29, S166-S174.

28. E. Delnoij, J. Westerweel, N. G. Deen, J. A. M. Kuipers, and W. P. M. van Swaaij, Chem. Eng. Sci., 1999, 54, 5159-5171.

29. S. Devasenathipathy, J. G. Santiago, S. T. Wereley, C. D. Meinhart, $90 \quad$ and K. Takehara, Exp. Fluids, 2003, 34, 504-514.

30. J. Westerweel, and F. Scarano, Exp. Fluids, 2005, 39, 1096-1110.

31. M. Raffel, C. E. Willert, S. T. Wereley, and J. Kompenhans, Particle Image Velocimetry: a practical guide, Springer, 2nd edn., 2007.

32. G. Minor, P. Oshkai, and N. Djilali, Meas. Sci. Technol., 2007, 18, L23-L28.

33. S. J. Kline, and F. A. McClintock, Mech. Eng., 1953, 75, 3-8.

34. S. Pennathur, Lab Chip, 2008, 8, 383-387.

35. P. Guillot, P. Panizza, J. Salmon, M. Joanicot, A. Colin, C.-H. Bruneau, and T. Colin, Langmuir, 2006, 22, 6438-6445.

100 36. H. Wong, C. J. Radke, and S. Morris, J Fluid Mech, 1995, 292, 95.

37. S. D. Hudson, Rheol. Acta, 2009, 49, 237-243.

38. B.-J. Jin, Y. W. Kim, Y. Lee, and J. Y. Yoo, J. Micromechanics Microengineering, 2010, 20, 035003.

39. J.-C. Baret, F. Kleinschmidt, A. El Harrak, and A. D. Griffiths, Langmuir, 2009, 25, 6088-6093.

40. J. D. Tice, H. Song, A. D. Lyon, and R. F. Ismagilov, Langmuir, 2003, 19, 9127-9133.

41. J. H. Xu, J. Tan, S. W. Li, and G. S. Luo, Chem. Eng. J., 2008, 141, 242-249. 